\title{
A finite-element numerical approach for modeling tsunamis
}

\author{
Stefano Tinti, Ivan Gavagni and Alessio Piatanesi \\ Dipartimento di Fisica, Settore di Geofisica, Università di Bologna, Italy
}

\begin{abstract}
A numerical scheme suitable for modeling tsunamis is developed and tested against available analytical solutions. The governing equations are the shallow water nonlinear nondispersive equations that are known to be appropriate for tsunami generation and propagation in coastal waters. The integration scheme is based on a finite-element space discretization, where the basic elements are triangles and the shape functions are linear. The time integration is a double step algorithm that is accurate to the second order in the time step $\Delta t$. The boundary conditions are pure reflectivity and complete transmissivity on the solid and open boundaries respectively and are implemented by modifying the time integration scheme in a suitable way. The model performance is evaluated by comparing the results with the analytical solutions in selected cases and is quite satisfactory, even when the grid has a coarse spatial resolution.
\end{abstract}

Key words analytical tests - boundary conditions finite-element - numerical model - shallow-water approximation - tsunami

\section{Introduction}

Tsunamis are rare events that are potentially capable of producing severe damage. Experimental data on such waves in the open ocean and in coastal waters are rare and mostly occasional, in the absence of a systematic observational network. Even large tsunamis that have occurred recently suffer from insufficient instrumental recording, though the destruction they caused on the coasts was such that they triggered timely post-event field surveys to determine accurately wave run-up, inundation extent, coastal depositional and erosional pattern, etc. (see for example, Baptista et al., 1993; Yeh et al., 1993; Gonzales and Bernard, 1993 concerning respectively the Nicaragua 1992 tsunami, the Flores, Indonesia, 1992 tsunami and the Hokkaido, Japan, 1993 tsunami; see also the very recent eastern Java tsunami). As a result, numerical simulations of tsunamis are very important tools of fundamental and ap- plied research to complement the scarce observational data set and to help illuminate basic aspects of wave generation, propagation and impact against the coast. This paper is devoted to illustrating and discussing a numerical approach that is based on the shallow-water fully nonlinear approximation suitable for studying tsunamis produced in coastal regions. The discretization in space is performed by means of a finite-element technique, while the integration in time is accomplished by a double time-step algorithm as will be briefly highlighted in the next sections. The approach is tested against analytical solutions of linear as well as non-linear shallow-water approximation for simple geometries of the water basin, which permit us to evaluate the accuracy of the integration scheme.

\section{The basic model}

Numerical simulations of tsunamis are commonly based either on the Boussinesq or on the nondispersive shallow water equations (Murty, 1977; Shuto, 1991), the latter being especially 
appropriate for tsunamigenesis occurring in coastal waters. In this paper we will adopt the shallow water approximation of the NavierStokes equations that is valid when the characteristic lengths of the water waves are much longer than the typical water depth. If we denote by $\zeta=\zeta(x, y, t), u=u(x, y, t), v=v(x, y, t)$ respectively the water elevation above the mean sea level, and the horizontal components of the water particle along the axes $x$ and $y$, the basic set of equations is written in the following form:

$$
\left\{\begin{array}{l}
\partial_{t} \zeta=-\partial_{x}(D u)-\partial_{y}(D v) \\
\partial_{t} u=-g \partial_{x} \zeta-u \partial_{x} u-v \partial_{y} u-C_{f} u V / D+f v \\
\partial_{t} v=-g \partial_{y} \zeta-u \partial_{x} v-v \partial_{y} v-C_{f} v V I D-f u
\end{array}\right.
$$

In the above equations $h=h(x, y)$ is the water depth, $g$ is the gravity acceleration, $C_{f}=$ is the friction coefficient and $f$ is the Coriolis parameter, given by $f=2 \omega \sin \varphi(\varphi$ is the latitude, $\omega$ is the Earth's angular velocity). The following definitions have been further used:

$$
\begin{gathered}
D(x, y, t)=h(x, y)+\zeta(x, y, t) \\
V^{2}(x, y, t)=u^{2}(x, y, t)+v^{2}(x, y, t)
\end{gathered}
$$

It is easy to see that $D$ represents the instantaneous water depth, depending dynamically on the motion conditions. The first of eqs. (2.1) derives from the water mass conservation, while the other two arise from the momentum conservation. The friction term is an experimental law expressing the shear at the ocean bottom. It is a typical resistance term subtracting energy from waves, and becomes important particularly over long time scales. Variants of the above approximation can be found in the literature: one that is often used adopts the flow components $Q_{x}=h u$ and $Q_{y}=h v$ rather than the water velocities (e.g. Liu et al., 1993). The eqs. (2.1) must be complemented by the boundary and the initial conditions, that as in all wave propagation problems should be mutually consistent in order to garantee the existence of a solution. These conditions will be discussed later on, while our attention is focussed next on the space discretization of the wave system (2.1).

The solution of the governing equations set is searched for in a finite basin, $\Omega$, that is partitioned into a number $M$ of polygonal nonoverlapping elements $\Omega_{k}$, the resulting grid having $N$ nodes. A basic idea for this approach is that within each element $\Omega_{k}$ any regular function $f(x, y, t)$ is approximated in terms of the values $f_{r}^{k}(t)$ that the function assumes at the nodes $r$ belonging to the element by means of a linear superposition of suitable shape functions, $S_{r}^{k}(x, y)$, depending solely on the geometry

$$
\begin{gathered}
f(x, y, t) \simeq f^{k}(x, y, t)= \\
=\sum_{r} S_{r}^{k}(x, y) f_{r}^{k}(t) \quad \text { in } \Omega_{k}
\end{gathered}
$$

Here the approximating function $f^{k}(x, y, t)$ is defined in an implicit manner. If we add the conditions that $S_{r}^{k}(x, y)=1$ at the node $r$ of $\Omega_{k}$ and be identically zero at all other nodes of the same element $k$, it follows that $f(x, y, t)=$ $=f^{k}(x, y, t)=f_{r}^{k}(t)$ at any node $r$. Now, if the solutions $\zeta(x, y, t), u(x, y, t)$ and $v(x, y, t)$ are similarly approximated by means of the above expression (2.4), the problem is simplified in that the only unknowns to determine are the nodal values of these functions, say $\zeta_{q}(t), u_{q}(t)$ and $v_{q}(t)$ where $q=1, \ldots, N$ is the index denoting the node.

A further basic idea is that the system of differential eqs. (2.1) is converted into a set of integral equations, by means of the following reasoning. If $\zeta, u$ and $v$ satisfy the eqs. (2.1), then they must identically fullfil the following integral form: 


$$
\left\{\begin{aligned}
\int_{\Omega} \zeta^{\star} \partial_{t} \zeta d \Omega= & -\int_{\Omega} \zeta^{\star} u \partial_{x} D d \Omega-\int_{\Omega} \zeta^{\star} D \partial_{x} u d \Omega- \\
& -\int_{\Omega} \zeta^{\star} v \partial_{y} D d \Omega-\int_{\Omega} \zeta \zeta^{\star} D \partial_{y} v d \Omega \\
\int_{\Omega} u^{\star} \partial_{t} u d \Omega= & -g \int_{\Omega} u^{\star} \partial_{x} \zeta d \Omega-\int_{\Omega} u^{\star} u \partial_{x} u d \Omega- \\
& -\int_{\Omega} u^{\star} v \partial_{y} u d \Omega+\int_{\Omega} f u^{\star} v d \Omega- \\
& -\int_{\Omega}^{u^{\star}} C_{f} \frac{u V}{D} d \Omega \\
\int_{\Omega} v^{\star} \partial_{t} v d \Omega= & -g \int_{\Omega} v^{\star} \partial_{y} \zeta d \Omega-\int_{\Omega} v^{\star} u \partial_{x} v d \Omega- \\
& -\int_{\Omega} v^{\star} v \partial_{y} v d \Omega-\int_{\Omega} f v^{\star} u d \Omega- \\
& -\int_{\Omega} v^{\star} C_{f} \frac{v V}{D} d \Omega
\end{aligned}\right.
$$

where $\zeta^{\star}, u^{\star}$ and $v^{\star}$ are arbitrary functions defined in the domain $\Omega$. Let us now take a set of independent Green functions $G_{i}(x, y) i=$ $=1, \ldots, N$, such that $G_{i}(x, y)=1$ at the node $i$ and $G_{i}(x, y)=0$ at all other nodes. If we pose $\zeta^{\star}=u^{\star}=v^{\star}=G_{i}(x, y)$ in the above integral system (2.5) and make use of the approximation (2.4), we obtain a number of $3 N$ conditions for the $3 N$ unknowns $\zeta_{q}(t), u_{q}(t)$ and $v_{q}(t)$, that can be solved by algebraic manipulations. For example, let us consider the left hand side integral in the first equation of the system (2.5). It can be firstly written in terms of the approximating functions

$$
\begin{gathered}
\int_{\Omega} \zeta^{\star}(x, y) \partial_{t} \zeta(x, y, t) d \Omega \simeq \\
\simeq \sum_{k} \int_{\Omega_{k}} G_{i}^{k}(x, y) \partial_{t} \zeta^{k}(x, y, t) d \Omega_{k}
\end{gathered}
$$

and then, on making use of the shape functions $S_{r}^{k}(x, y)$, it is transformed into

$$
\begin{gathered}
\int_{\Omega} \zeta^{\star}(x, y) \partial_{t} \zeta(x, y, t) d \Omega \simeq \\
\simeq \sum_{k} \int_{\Omega_{k}} \sum_{r s} S_{r}^{k}(x, y) \delta_{r i}^{k} S_{s}^{k}(x, y) \dot{\zeta}_{s}(t) d \Omega_{k}
\end{gathered}
$$

where $\delta_{r i}^{k}$ is the Kronecker delta and the internal double sum in $r$ and $s$ extends over all nodes belonging to $\Omega_{k}$. Here the symbol $\dot{\zeta}_{s}(t)$ is used to denote the time derivative of the function $\zeta_{s}(t)$. Since the shape functions are known, the integrals over each element $\Omega_{k}$ can be computed in a closed form and the eq. (2.7) can be easily given the following expression:

$$
\int_{\Omega} G_{i}(x, y) \partial_{t} \zeta(x, y, t) d \Omega \simeq \sum_{q} K_{i q} \dot{\zeta}_{q}(t)
$$

The above coefficients $K_{i q}$ have the form:

$$
K_{i q}=\sum_{k} \int_{\Omega_{k}} S_{i}^{k}(x, y) S_{q}^{k}(x, y) d \Omega_{k}
$$

and identify a linear symmetric operator $K$ in the space $R^{N}$ acting on the vector $\widetilde{\zeta}(t)$ with components $\zeta_{q}(t)$. Likewisely we can proceed to discretise in space all other integrals of the system (2.5), that in this way is converted into a system of equations in the unknown vectors $\widetilde{\zeta}(t), \tilde{u}(t)$ and $\widetilde{v}(t)$ depending only upon the time $t$ :

$$
\left\{\begin{aligned}
K \dot{\tilde{\zeta}}= & -\Psi_{x}(\tilde{u}) \tilde{\zeta}-\Psi_{x}(\tilde{D}) \tilde{u}-\Psi_{y}(\tilde{v}) \tilde{\zeta}-\Psi_{y}(\tilde{D}) \tilde{v}- \\
& -\bar{\Psi}_{x}(\tilde{h}) \tilde{u}-\bar{\Psi}_{y}(\tilde{h}) \tilde{v} \\
K \dot{\tilde{u}}= & -g P_{x} \tilde{\zeta}-\Psi_{x}(\tilde{u}) \tilde{u}-\Psi_{y}(\tilde{v}) \tilde{u}+K f \tilde{v}- \\
& -\Phi(\tilde{V}, \tilde{D}) \tilde{u} \\
K \dot{\tilde{v}}= & -g P_{y} \tilde{\zeta}-\Psi_{x}(\tilde{u}) \tilde{v}-\Psi_{y}(\tilde{v}) \tilde{v}-K f \tilde{u}+ \\
& +\Phi(\tilde{V}, \tilde{D}) \tilde{v}
\end{aligned}\right.
$$

Here operators are designated by capital letters, whereas vectors are overlined by a tilde. The subscripts $x$ and $y$ are used to indicate that the operator derives from a space derivative in the direction of the corresponding axis. The nota- 
tion $\Psi_{x}(\tilde{u})$ is suggestive that the operator is not constant, but depends upon the values of the unknown vector $\widetilde{u}(t)$, with the consequence that the operator itself is unknown. Such operators are present in all equations of the system (2.10) and this is the patent expression of the nonlinearity of the original problem (2.1).

In order to illustrate the time discretization it is convenient to put the above system in the more compact form

$$
A \dot{\tilde{\alpha}}(t)=B(\tilde{\alpha}) \tilde{\alpha}(t)
$$

where $\tilde{\alpha}(t)$ is a $3 N$-component vector defined as:

$$
\tilde{\alpha}=\left(\begin{array}{c}
\tilde{\zeta} \\
\tilde{u} \\
\tilde{v}
\end{array}\right)
$$

and consonantly the operators $A$ and $B$ defined in the space $R^{3 N}$ are given by

$$
\begin{gathered}
A=\left(\begin{array}{ccc}
K & 0 & 0 \\
0 & K & 0 \\
0 & 0 & K
\end{array}\right) \\
B=\left(\begin{array}{ccc}
-\Psi_{x}(\widetilde{u})-\Psi_{y}(\widetilde{v}) & -\Psi_{x}(\widetilde{D})-\bar{\Psi}_{x}(\widetilde{h}) & -\Psi_{y}(\widetilde{D})-\bar{\Psi}_{y}(\widetilde{h}) \\
-g P_{x} & -\Psi_{x}(\widetilde{u})-\Psi_{y}(\widetilde{v})-\Phi(\widetilde{V}, \widetilde{D}) & K f \\
-g P_{y} & -K f & -\Psi_{x}(\widetilde{u})-\Psi_{y}(\widetilde{v})-\Phi(\widetilde{V}, \widetilde{D})
\end{array}\right)
\end{gathered}
$$

It is apparent that the operator $A$ is a block matrix consisting of identical, diagonal and symmetrical blocks $K$ acting in $R^{N}$. Let us now suppose that the solution $\widetilde{\alpha}_{n}=\widetilde{\alpha}\left(t_{n}\right)$ at the time $t_{n}=t_{0}+n \Delta t$ is known and let us try to determine the solution at the subsequent time step $t_{n+1}$. If we pose $\tilde{x}_{n}=B\left(\tilde{\alpha}_{n}\right) \tilde{\alpha}_{n}$ the differential eq. (2.11) simplifies into

$$
A \dot{\tilde{\alpha}}_{n}=\tilde{x}_{n}
$$

After differentiating this equation with respect to the time, we obtain the approximate expression

$$
A \ddot{\tilde{\alpha}}_{n} \simeq \frac{2}{\Delta t}\left(\tilde{x}_{n+1 / 2}-\tilde{x}_{n}\right)
$$

A Taylor expansion of the unknown vector $\tilde{\alpha}$ up to the second order in $\Delta t$ leads to the approximation

$$
\tilde{\alpha}_{n+1} \simeq \tilde{\alpha}_{n}+\Delta t \dot{\tilde{\alpha}}_{n}+\frac{\Delta t^{2}}{2} \ddot{\tilde{\alpha}}_{n}
$$

Combining the eqs. (2.15) and (2.16) in the expansion (2.17), the following two-step resolving scheme is obtained

$$
\begin{aligned}
\tilde{\alpha}_{n+1 / 2} & =\tilde{\alpha}_{n}+\frac{\Delta t}{2} A^{-1} \tilde{x}_{n} \\
\tilde{x}_{n+1 / 2} & =B\left(\tilde{\alpha}_{n+1 / 2}\right) \tilde{\alpha}_{n+1 / 2} \\
\tilde{\alpha}_{n+1} & =\tilde{\alpha}_{n}+\Delta t A^{-1} \tilde{x}_{n+1 / 2}
\end{aligned}
$$

It can be appreciated that this procedure requires solely the inversion of the matrix $A$ that in turn implies the only inversion of $K$.

The initial and boundary conditions are necessary in order to identify and compute the solution of the wave problem (2.1). In tsunami computations it is usual to give the initial condition by specifying the initial elevation of the water above the mean sea level $\zeta\left(x, y, t_{0}\right)=$ $\zeta_{0}(x, y)$ and by assuming that the initial velocity field be identically zero, i.e. $u\left(x, y, t_{0}\right)=$ $v\left(x, y, t_{0}\right)=0$. This is adequate when the generation process is instantaneous, that is when it occurs over a time scale $(\sim 1 \mathrm{~s})$ much shorter than the tsunami typical periods $\left(\sim 10^{2}-10^{3} \mathrm{~s}\right)$. 
Therefore it is applicable to the case of a tsunami generated by a sizable submarine earthquake, while the theory should be slightly accomodated to deal with slower generative processes such as submarine landslides or extremely large earthquakes where the fault rupturing time is a significant parameter. And this is commonly accomplished by introducing a moving source with known kinematics (Harbitz, 1991; Mader et al., 1993a,b). The conditions on the boundary are prescribed by differentiating between the coastal boundary and the open boundary. A pure reflective boundary is normally considered adequate for the coastline, unless specific studies on flooding, inundation or impact on coastal structures are to be carried out. The pure reflectivity condition is expressed by posing the velocity component normal to the boundary equal to zero. On the ocean side a pure transmission condition is to be imposed, letting the wavefronts cross the boundary without any artificial modification. This undisturbed propagation is more difficult to obtain, but it is approached by imposing a relation of outgoing wave for the wave component normal to the boundary. Therefore these conditions can be expressed in the following way:

$$
\begin{array}{ll}
\vec{v} \cdot \vec{n}=0 & \text { on the solid boundary } \\
\vec{v} \cdot \vec{n}=\frac{g}{c} \zeta & \text { on the open boundary }
\end{array}
$$

where $\vec{v}$ and $\vec{n}$ are the horizontal velocity vector and the outward normal vector respectively, while $c$ is defined as $c=\sqrt{g h}$ representing the local phase velocity of the waves in the linear shallow-water approximation. If we call $\widetilde{\alpha}_{c}$ a vector in the space $R^{3 N}$ satisfying the boundary conditions $(2.19 \mathrm{a}, \mathrm{b})$, let us define by $C$ the

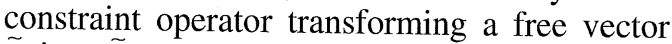
$\widetilde{\alpha}$ into $\widetilde{\alpha}_{c}$, that is:

$$
\tilde{\alpha}_{c}=C \tilde{\alpha}
$$

In virtue of the above relation, the boundary conditions are implemented in our model by adding two supplementary steps to the resolv-

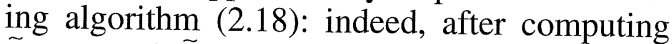
$\tilde{\alpha}_{n+1 / 2}$ and $\tilde{\alpha}_{n+1}$, these are transformed into their respective constrained counterparts by means of the operator $C$. We observe that the incorporation of constraints in the time-integration scheme is based firmly on the theory in the linear approximation (Piatanesi, 1994) and further that it has the remarkable advantage of requiring the inversion of the only matrix $K$. On the contrary, a model imposing the constraints in the space discretisation stage will give rise to a matrix $A$ formed by diagonal blocks, say $K_{\zeta}, K_{u}$ and $K_{v}$, not equal, but differing from each other (see eq. (2.13)). And accordingly, the inversion of $A$ required in the eq. (2.18) would imply the inversion of 3 different matrices instead of one. Our method therefore saves computer storage and time.

\section{Numerical tests}

The model schematised in the previous section has been developed on meshes consisting of triangular elements where the mesh nodes coincide with the triangle vertexes. The partition of the domain $\Omega$ into triangles has the advantage that they have the simplest geometrical shape and that nevertheless the grid is highly flexible and adaptable to any kind of ocean basin configuration. In particular, irregular coastlines can be modeled in a suitable way (Tinti and Mulargia, 1983; Werner and Linch, 1987; Greenberg et al., 1993), whereas this cannot be achieved by finite-difference models that are generally conceived for regular-cell meshes based on cartesian, polar or spherical coordinate systems. The shape functions $S_{r}^{k}(x, y)$ used in our model are linear functions: practically they represents plane surfaces with unit value at the vertex $r$ of the triangle $\Omega_{k}$ and vanishing at the other two vertexes. This choice implies that all physical fields, namely $\zeta, u$ and $v$, resulting from a superposition of shape functions (see eq. (2.4)), are represented as well by plane surfaces within each triangular element. The time step $\Delta t$ used in the algorithm (2.18) is taken according to the following 
(a)
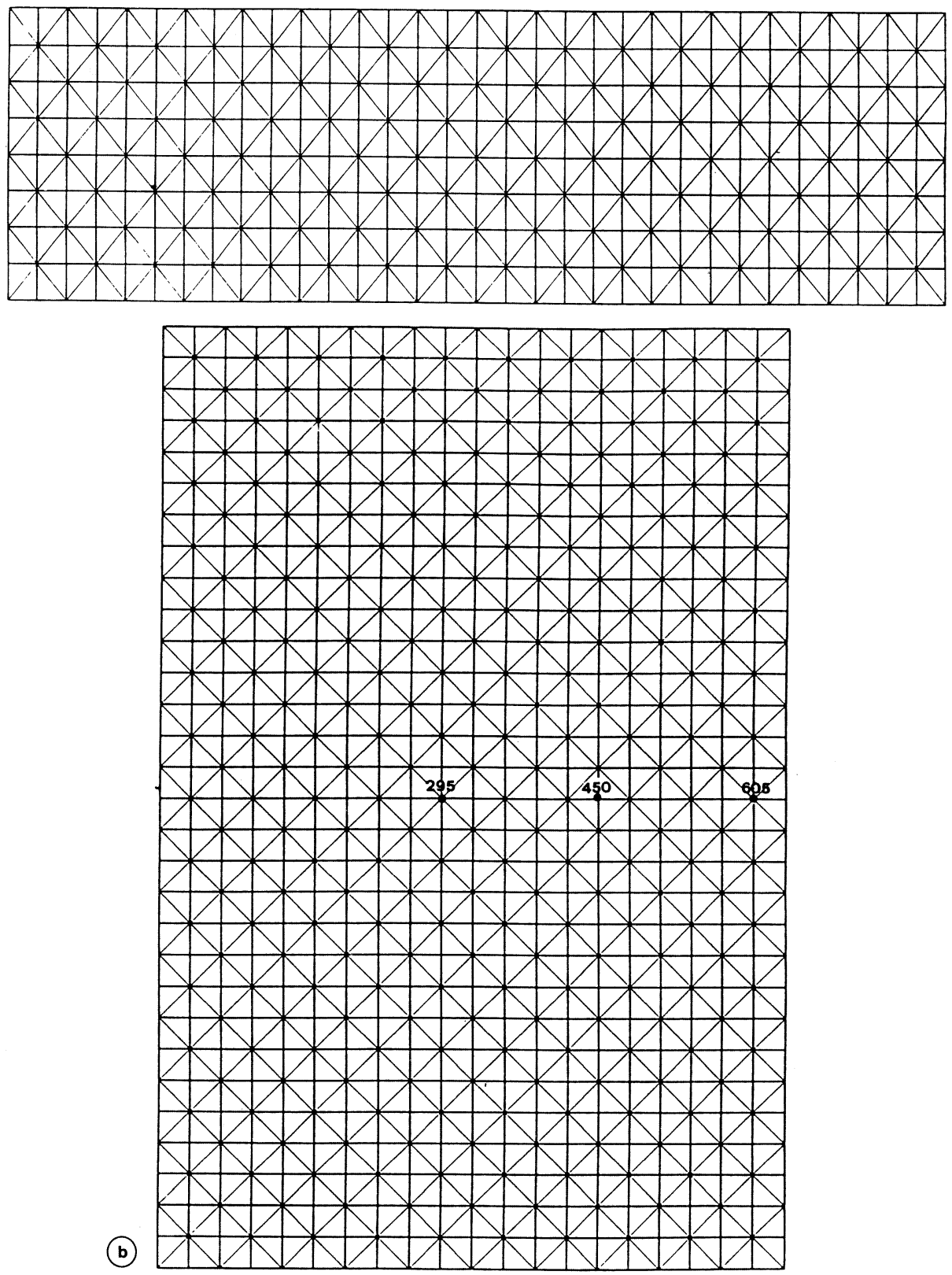

(b)

Fig. 1a,b. Sketch of the grids used in the paper. a) Rectangular channel: 512 equal triangles and 297 nodes. Channel length, width and depth are in the order $16 \mathrm{~km}, 5 \mathrm{~km}$ and $5 \mathrm{~m}$. The channel is open at the right and left boundaries. b) Rectangular grid $40 \mathrm{~km}$ long, $60 \mathrm{~km}$ wide with 1200 elements and 651 nodes. The basin is closed by a vertical reflecting wall on the right and open at the other boundaries. The floor depth is $400 \mathrm{~m}$ for the uniform bottom case. Solid circles indicate the nodes where time histories are calculated and shown for the variable bottom case (see fig. 7). 
criterion. Let us introduce the characteristic time $\Delta t_{k}$ for the element $\Omega_{k}$ that is the mean time taken by a wave to cross the element and that can be estimated by $\Delta t_{k}=L_{k} / c_{k}$. Here $L_{k}$ is the characteristic element length and $c_{k}$ is the local wave velocity. If we designate by $\Delta T$ the smallest of all characteristic times $\Delta t_{k}$, in our numerical experiments we have taken a time step $\Delta_{t}=\gamma \Delta T$ where the coefficient $\gamma$ is in the range $0.2-0.5$, which is an empirical good compromise between time efficiency and scheme stability.

The model has been tested against several analytical solutions computed in cases that, though simple, are equally significant to show the model performance. Some of these cases will be presented in this section. The most elementary example is the rectangular channel of uniform depth shown in fig. 1a that has been divided into $M=512$ equal triangles corresponding to $N=297$ nodes. The channel is bounded by two vertical reflective longer walls, while the shorter sides on the left and on the right are a distance $L=16 \mathrm{~km}$ apart and are open, permitting the waves to pass away. The channel is taken to be very shallow $(h=5 \mathrm{~m})$, causing the waves to proceed quite slowly, remaining in the channel for a sufficiently long time. The basin is excited by an initial water bulge that is symmetric and transversally uniform, depending only on the longitudinal coordinate $x$. Figure 2 shows the travelling waves along the longitudinal axis of the channel computed through the linear version of the numerical model with time step $\Delta t=5 \mathrm{~s}$ and compares them with the analytical solution that is easily calculated in 1D space (see Appendix for details). Though the case is critical since the grid resolution has been deliberately taken poor with respect to the assumed initial disturbance, that is described only by a few nodes per dominant wavelength, there is no visible difference between the two solutions during the initial wave splitting and propagation in the internal waters. Even the waves crossing the open boundaries are computed correctly. Later some residual noise that is tendentially growing with time is apparent in the numerical solution, but it does not perturb the wave evolution, since it becomes relevant only after the waves leave the channel.

The second example regards a $2 \mathrm{D}$ propagation in a flat bottom $400 \mathrm{~m}$ deep rectangular basin that is $40 \mathrm{~km}$ long ( $x$-axis) and $60 \mathrm{~km}$ wide ( $y$-axis). The grid is formed by 1200 equal triangles corresponding to 651 nodes that is depicted in fig. 1b. The boundary on the right is closed, while all others are open. The initial disturbance is an axisymmetric bulge centered in the mid-point of the right boundary of the basin and is given in fig. 3. The analytical solution in the linear approximation (see Appendix) is shown side by side with the numerical solution at different time steps in figs. $4 a-c$ where contour plots of the water elevation are visible. It can be observed that the numerical model works quite well, in that it is able to compute satisfactorily the outgoing circular wavefronts, even though the grid geometry is not specifically conceived for a polar coordinate system. Even in this case, after the main wave system leaves the domain, some minor residual noise is left, which, however, does not prejudice the overall wave evolution. A more critical example is run by using the same initial excitation on a similar basin, having however a variable depth bottom. The variable bathymetry that is shown in fig. 5 is axisymmetric as well, since this property permits the computation of the linear solution by analytical means (see Appendix). The initial water rise propagates radially changing profile and wavelength as it proceeds towards deeper waters, and all these features are properly simulated by the numerical model as is apparent from fig. 6, where analytical and numerical solutions are juxtaposed. Analogously the timehistories computed via both methods for some nodes located on the central axis of the basin are given together in fig. 7.

The last example dealt with here is the nonlinear propagation of a trough along a channel. The same channel displayed in fig. 1a, but with a deeper floor $(h=20 \mathrm{~m})$, is forced by a transversally uniform disturbance, entering from the left side of the channel, on which both the water elevation and velocity are prescribed as given functions of time. The trough is advancing rightwards and deforms during its 

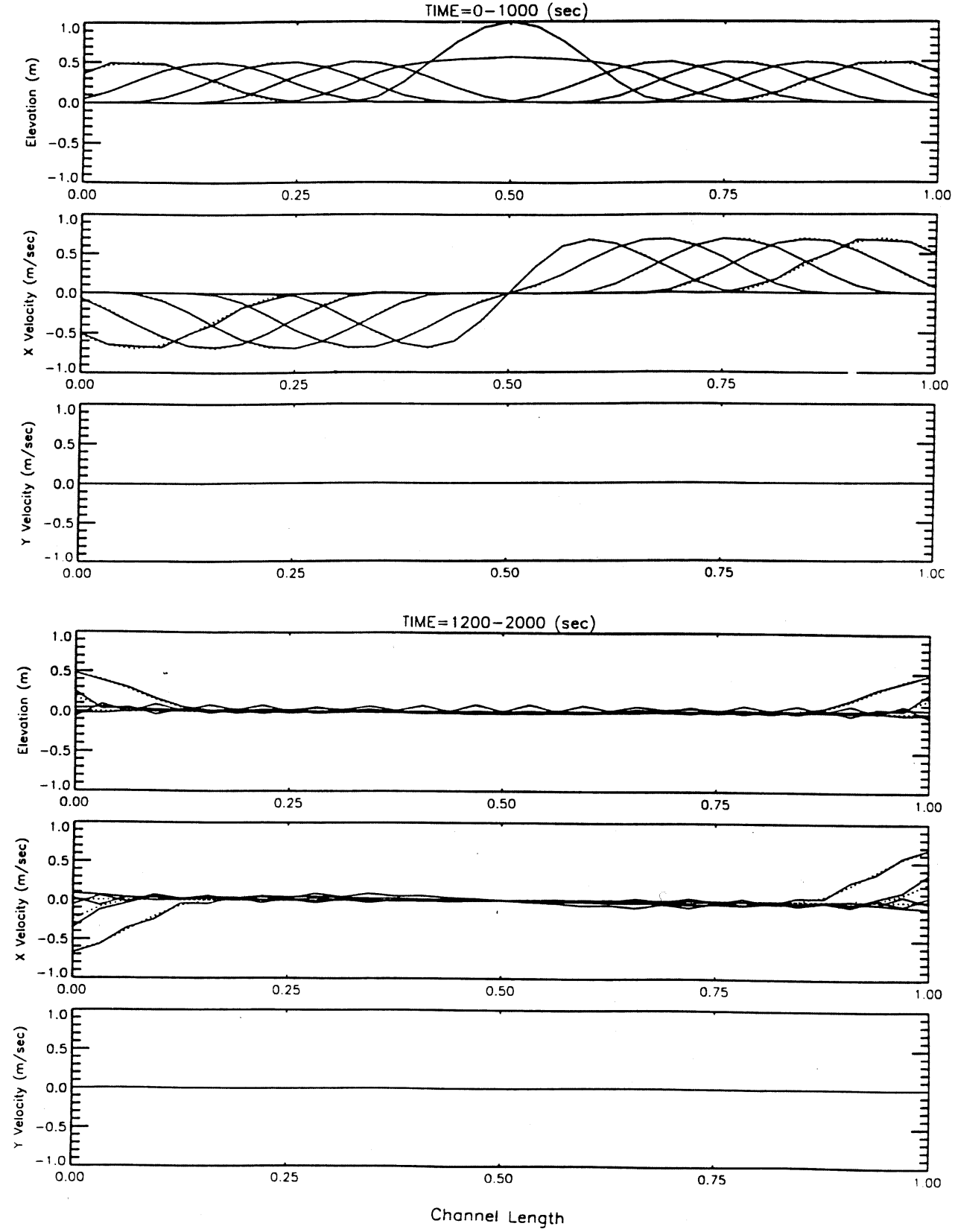

Fig. 2. Propagation of the initial bulge along the central axis of the channel at different times. Distances are normalized with respect to the channel length. The wave elevation progresses symmetrically towards the channel right and left ends, while the velocity $u$ is antisymmetric and $v$ is identically zero. Numerical results (solid line), computed with $\Delta t=5 \mathrm{~s}$, are compared with the analytical solution (dotted line) any $200 \mathrm{~s}$ in the time window 0-2000 s. 


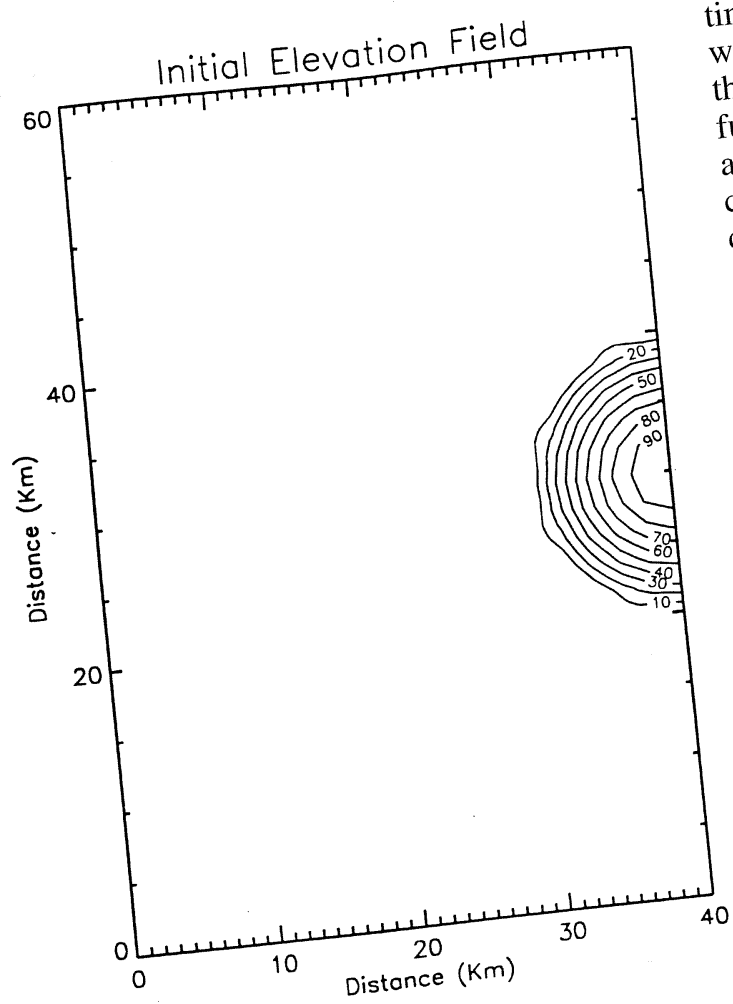

Fig. 3. Initial water disturbance in $\mathrm{cm}$ for the cases Filustrated in the next figs. $4 a-c$ and 6 . It is an axisymmetric bulge with the maximum height of $1 \mathrm{~m}$. propagation as the precipuous local phase velocity nonlinear terms. Since the function of the local water is an increasing fum of the trough is slower depth $D$, the bottom of thear-walls: consethan its ascending frons smoother, whereas the quently the front tureer and steeper until the back becomes limit is reached. The analytical wave breaking calculated by means of the theory of the characteristics (see Appendix) and is compared with the numerical solution along the central axis of the channel in fig. 8. The trough profile is reproduced satisfacto channel until the during its motion alon crossing the right side. wave leaves the basin cright discrepancy is apparObserve that some slight discrepancy is anderes-
ent since the numerical results tend to under 

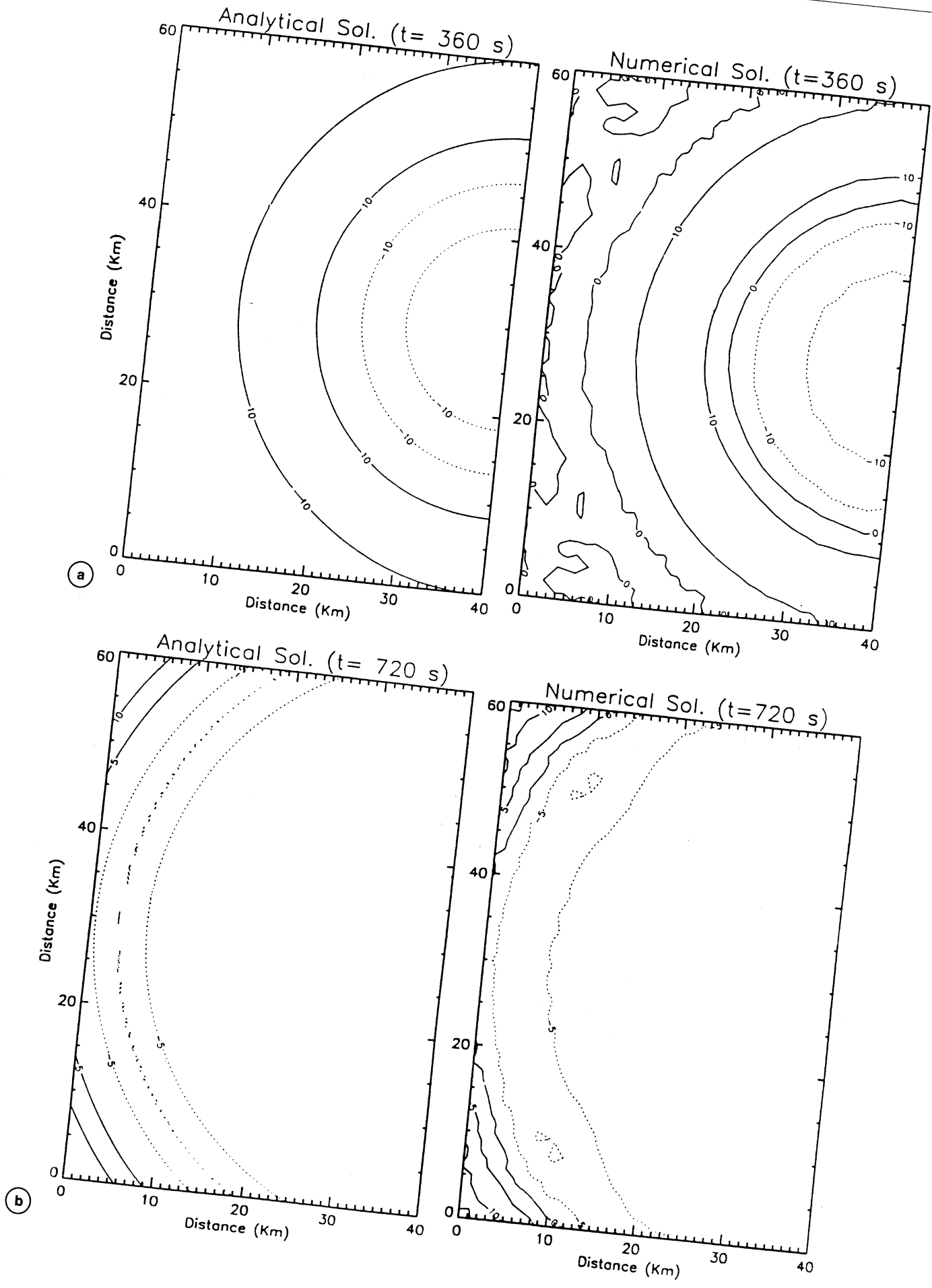

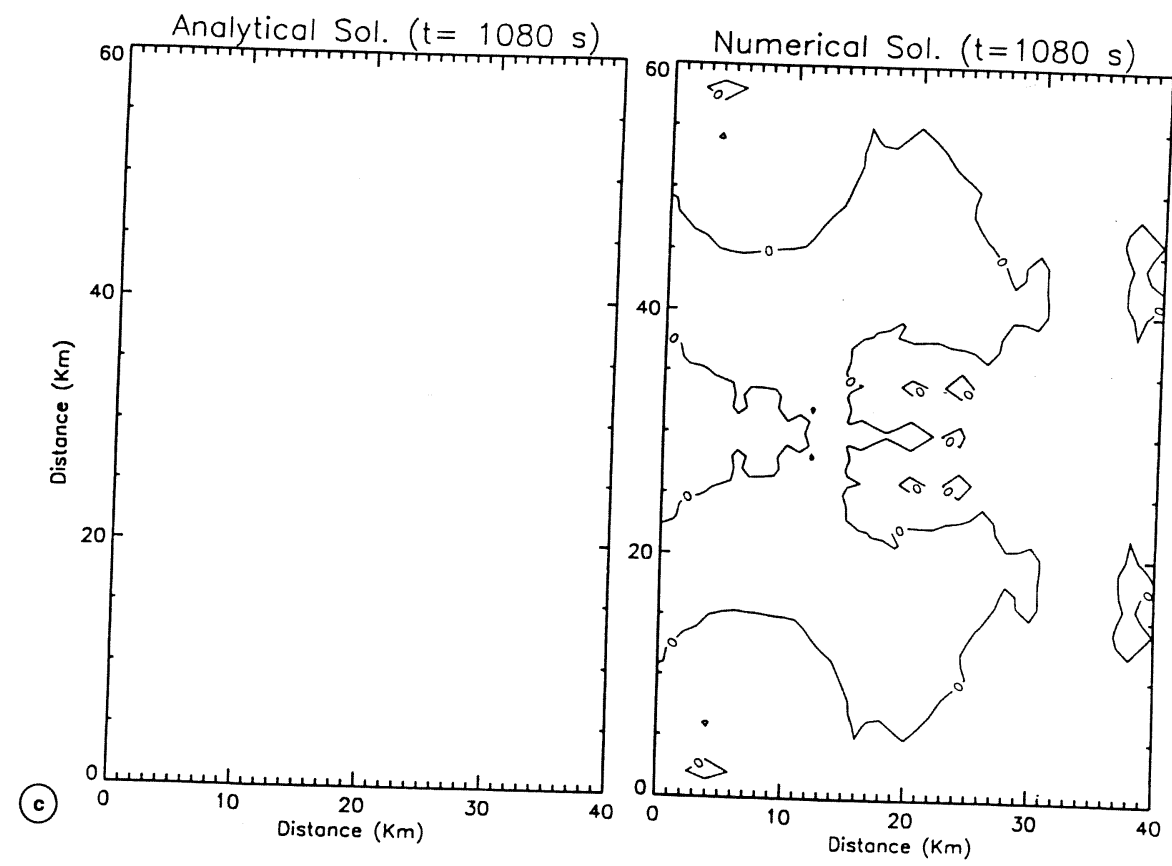

Fig. 4a-c. Water elevation fields at different times: a), b) and c) correspond to times $360 \mathrm{~s}, 720 \mathrm{~s}$ and $1080 \mathrm{~s}$ respectively. Numerical solutions (right) are compared with the analytical solutions (left). Irregularities in the contour lines are due to slight numerical noise and to contour plotting with poor resolu-
tion.

system. If, however, we have an interest in long time scale integration, a feasible way to control noise growing is to add a suitable smoothing algorithm in the time integration scheme (2.18) (Gavagni, 1993). The nonlinear propagation of the trough computed by means of our model incorporating a smoothing stage is presented in fig. 9 that can be compared with the results shown in fig. 8 . The improvement is noticeable, though some coherent energy is still visible inside the basin after the passage of the main wave.

Fig. 5. Bathymetric map of the rectangular basin Contour levels are in meters. The map is based on the grid of fig. 1a,b and has therefore a resolution depending on the triangle size.

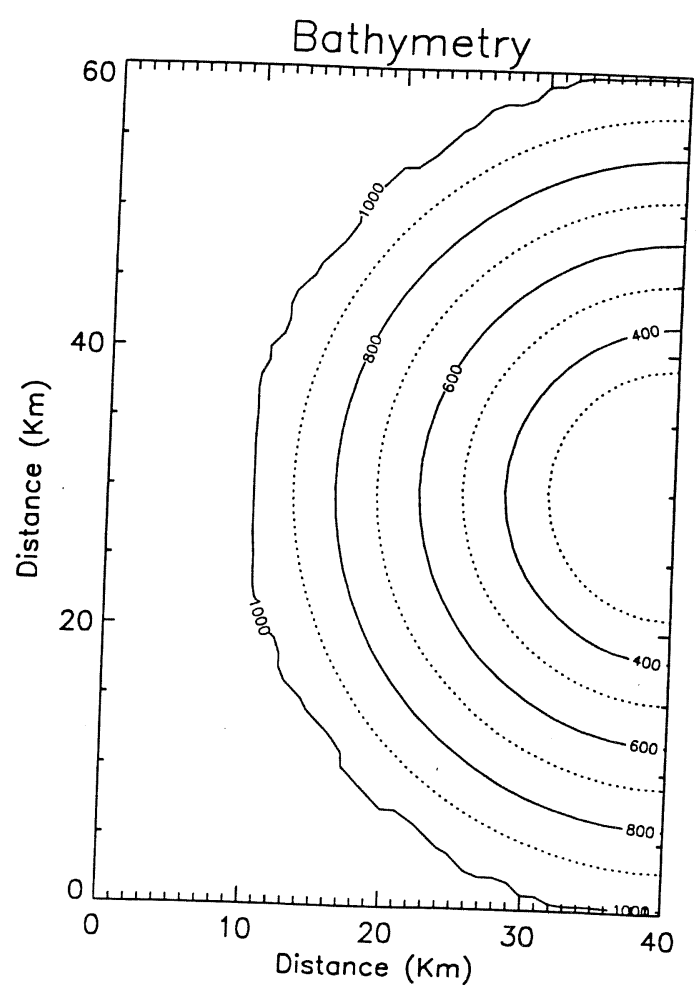



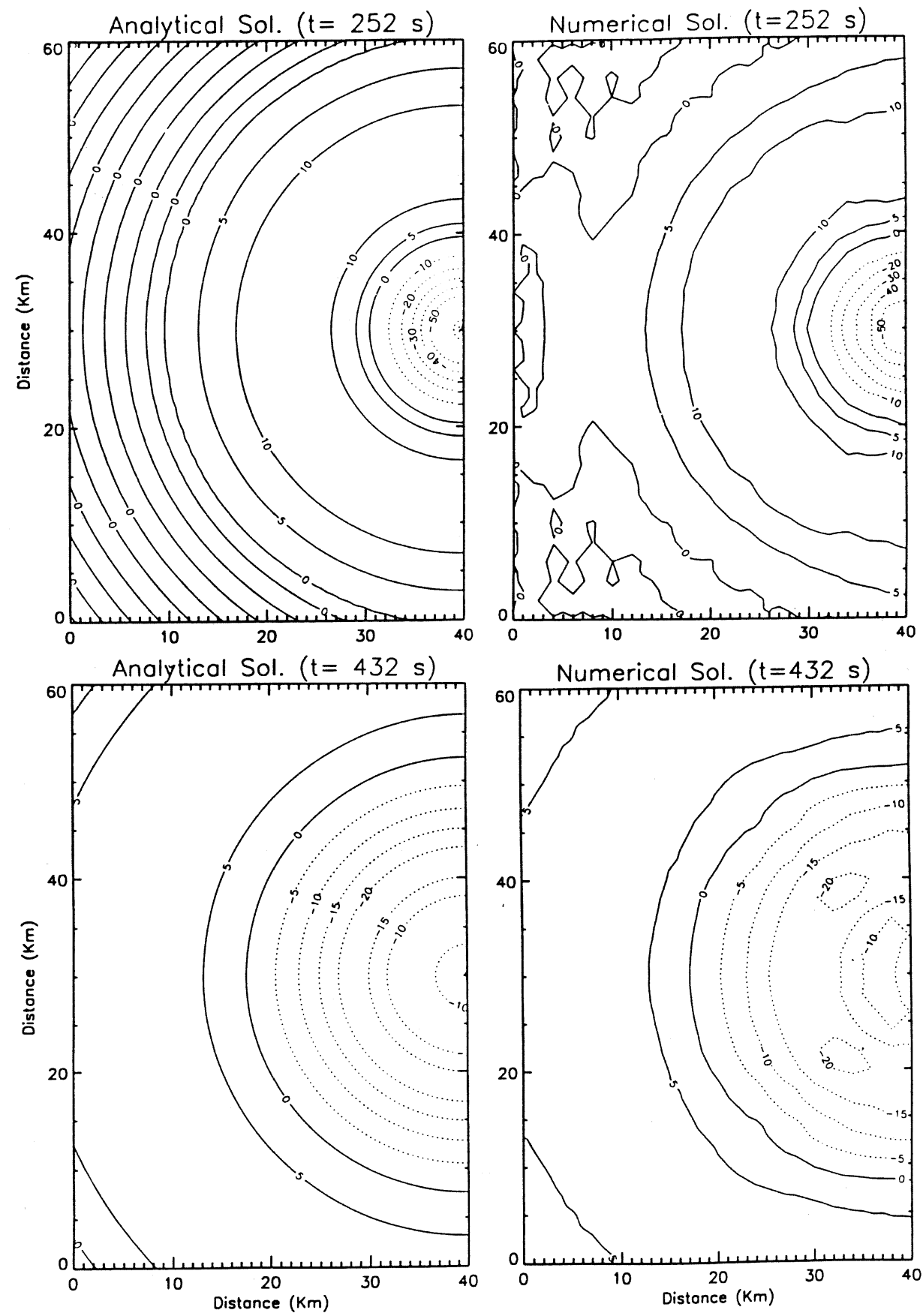

Fig. 6. Water elevation fields at different times computed for the variable depth basin. Remarks in the caption to fig. 4a-c also apply here. 

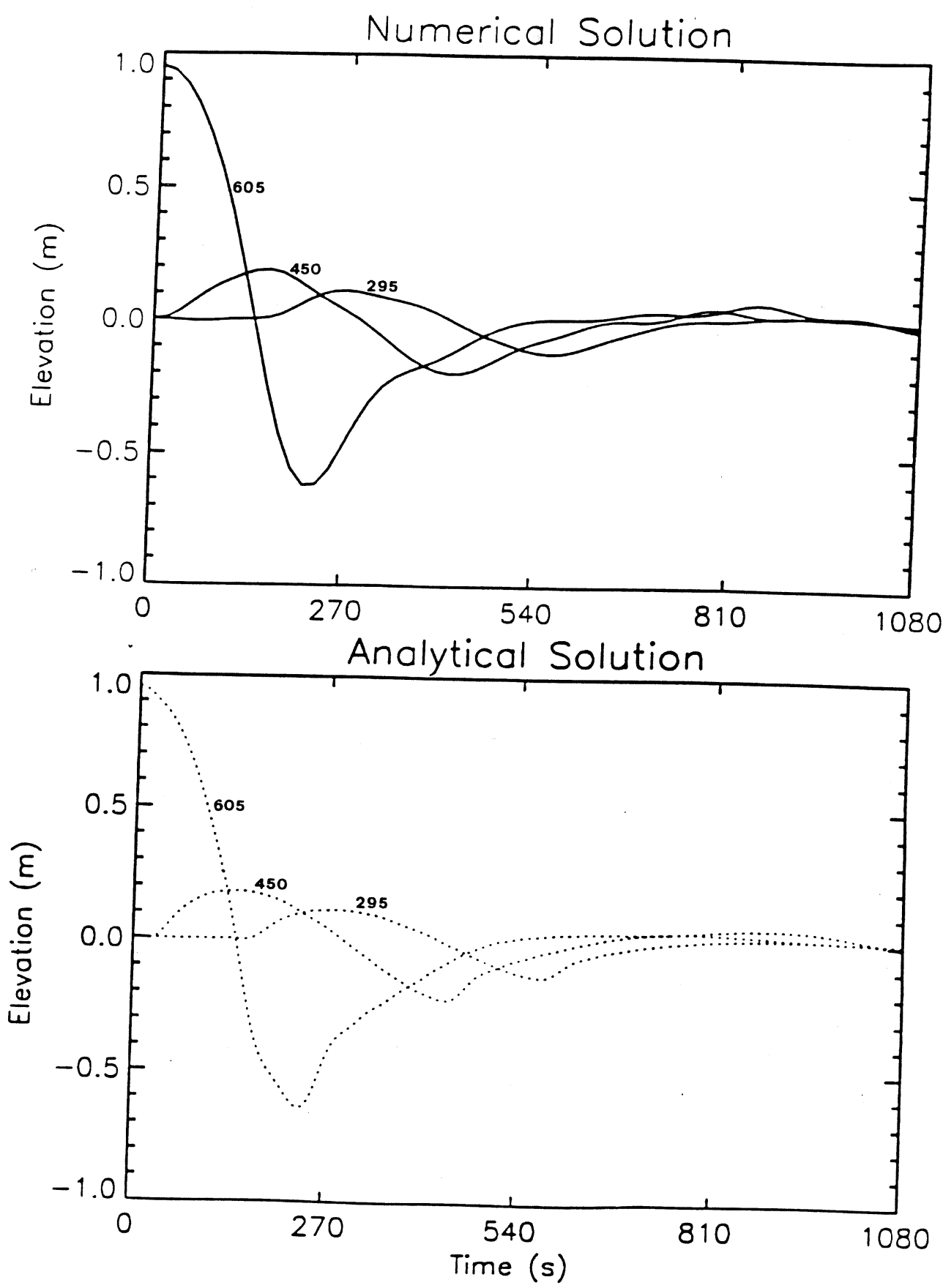

Fig. 7. Time histories calculated at the nodes shown on the grid of fig. 1a,b. Analytical solutions (dotted lines) and numerical results (solid lines) are shown. Nodes are marked by numbers, that are arranged in in-
creasing order from left to right in the grid. 

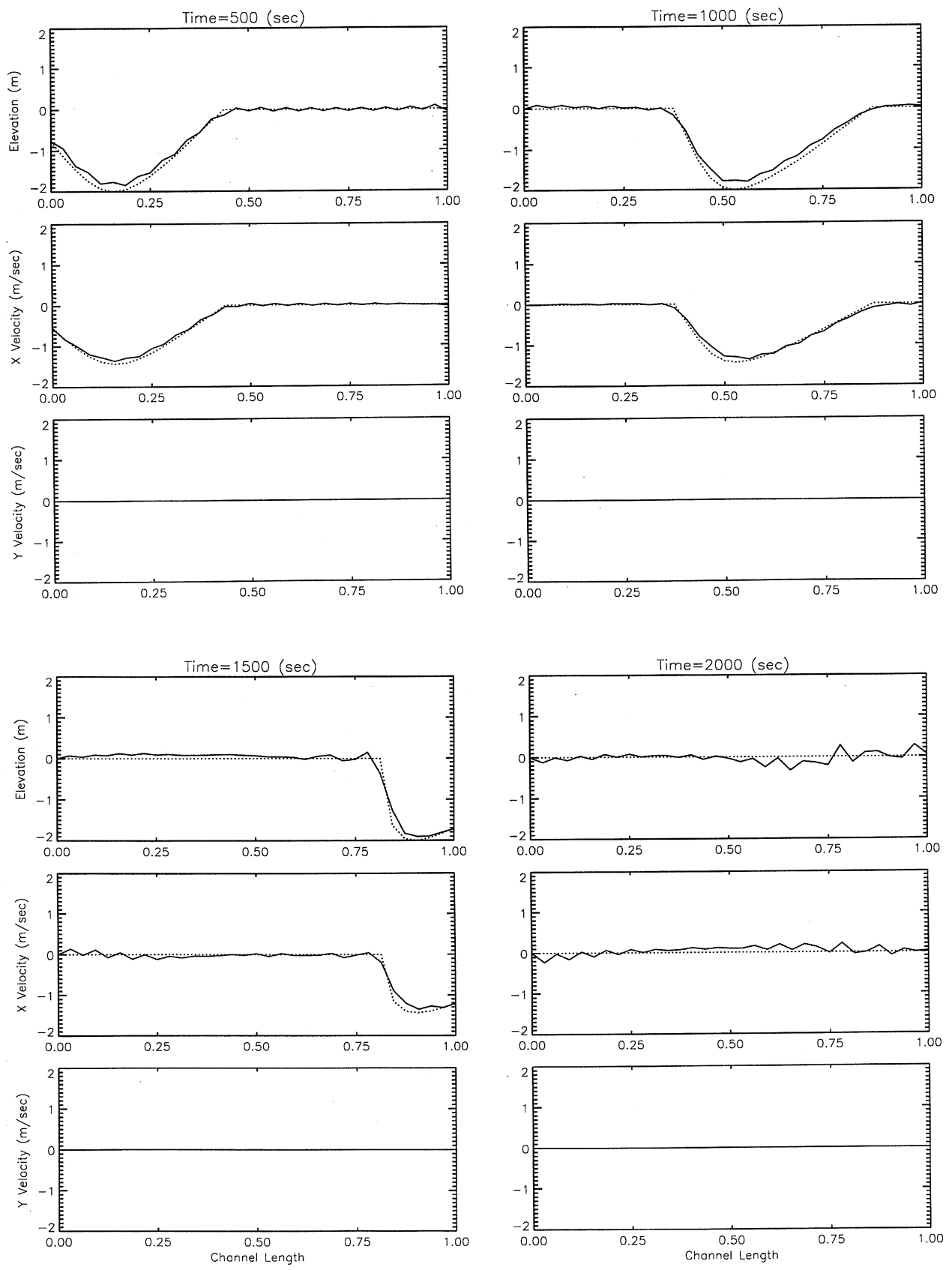

Fig. 8. Nonlinear propagation of a trough along the central axis of the channel of fig. 1a,b, with $20 \mathrm{~m}$ depth. The trough enters from the open left side and progresses towards the right changing shape. Numerical results (solid lines) can be compared with the theoretical solutions (dotted lines) computed by means of the theory of characteristics. 

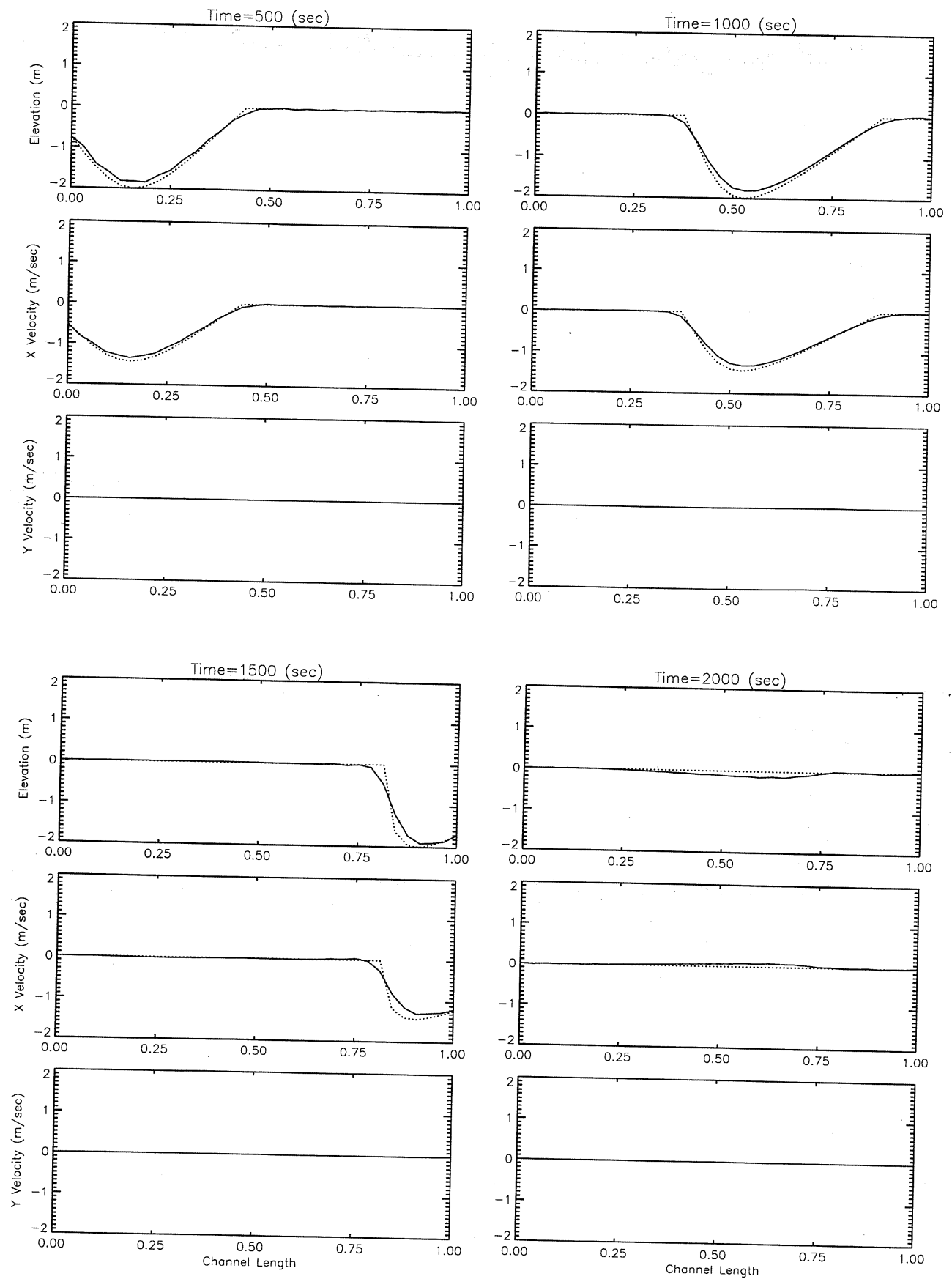
Fig. 9. Nonlinear propagation of the water depression as in fig. 8. Numerical profiles are computed by
incorporating a smoothing algorithm in the time integration scheme. 
The finite-element method we have illustrated here has been developed and tested with the main purpose of using it for numerical simulations of the Italian tsunamis. It is known that Italy and Greece are the two Mediterranean countries most exposed to the menace of tsunamis and the tsunami hazard for the Italian coasts has been given quantitative estimates through appropriate statistical methods (Tinti, 1991, 1993; Tinti et al., 1994). It is however essential to study the impact on the coasts of individual tsunamis, in order to make sensible progress in assessing local tsunami hazard and risk. Historical cases are often poorly documented, and therefore numerical simulations are indispensable means for tsunami research in Italy. Modeling of the major Italian tsunamis will be an important future evolution of our research.

\section{Acknowledgements}

This work has been partly financed by funds from MURST (the Italian Ministero dell'Università e Ricerca Scientifica e Tecnologica) and partly by means of funds from the European Community: EVCV-CT92-0175. The authors are indebt with Dr. C. Vannini for his help in computing and drawing the fields of the axisymmetric bulge propagating on a uniform as well as on a variable depth basin (see figs. 4a-c and 6).

\section{REFERENCES}

Baptista, A.M., G.R. Priest and T.S. Murty (1993): Field survey of the 1992 Nicaragua Tsunami, Marine Geodesy, 16, 169-203.

GavaGnI, I. (1993): Simulazioni numeriche di onde di maremoto mediante un programma di integrazione agli elementi finiti, Tesi di laurea, pp. 115 (in Italian).

Gonzales, F.I. and E.N. Bernard (1993): The 1993 Hokkaido-Nansei-Oki tsunami (abstract), EOS, Transactions, 1993 AGU Fall Meeting, 74, 349.

GreenberG, D.A., T.S. Murty and A. Ruffman (1993): A numerical model for the Halifax Harbor tsunami due to the 1917 explosion, Marine Geodesy, 16, 153-167.

Harbitz, C. (1991): Numerical simulation of slide generated water waves, Science of Tsunami Hazards, 9, 15-22.

Liu, P.L., S.B. Yoon, S.N. SEO and Y. CHo (1993): Numerical simulations of tsunami inundation at Hilo, Hawaii, in Proceedings of the IUGG/IOC International Tsunami Symposium, Wakayama, Japan, 257-269.

Mader, C.L., D.W. Moore and G.F. CArrier (1993a): Numerical tsunami source study - II, Science of Tsunami Hazards, 11, 81-92.

Mader, C.L., D.W. Moore and G.F. Carrier (1993b): Numerical tsunami propagation study - III, Science of Tsunami Hazards, 11, 93-106.

MurTy, T.S. (1977): Seismic sea waves - tsunamis, Bulletin 198 (Fisheries and Environment, Ottawa, Canada), pp. 337.

Piatanesi, A. (1994): Sviluppo e applicazione di un modello numerico di simulazione di onde di maremoto, Tesi di laurea, pp. 95 (in Italian).

Shuto, N. (1991): Numerical simulation of tsunamis - its present and future, in Tsunami Hazard, edited by E.N. Bernard (Kluwer Academic Publishers), 171-191.

Stoker, J.J. (1957): Water Waves, the Mathematical Theory with Applications (Interscience Publishers, Inc., New York), pp. 567.

TinTI, S. (1991): Assessment of tsunami hazard in the Italian seas, Natural Hazards, 4, 267-283.

TINTI, S. (1993): Evaluation of tsunami hazard in Calabria and Eastern Sicily, Italy, in Tsunamis in the World, edited by S.TINTI, Advances in Natural and Technological Hazards Research (Kluwer Academic Publishers, Dordrecht, The Netherlands), vol. 1, 141-157.

Tinti, S. and F. Mulargia (1983): Numerical simulation of the 1908 Messina tsunami: preliminary results, in Proceedings XVIII General Assembly of ESC, Akademie Wissenschaften der DDR, Potsdam, 407-411.

Tinti S. and C. VAnNini (1994): Theoretical investigation on tsunamis induced by seismic faults near ocean islands, Marine Geodesy (in press).

Tinti S., A. Maramai and P. Favali, (1994): The Gargano promontory: an important seismogenic-tsunamigenic area, Marine Geology, 17, 193-212.

WERNER, F.E. and D.R. LYNCH (1987): Field verification of wave equation tidal dynamics in the english channel and southern north sea, Advances Water Resources, 10, 115-130.

Yeh, H., F. Imamura, C. Synolakis, Y. Tsuji, P. Liu and S. SHI (1993): The Flores Island tsunamis, EOS, Transactions, 74, 369-373.

(received April 20, 1994; accepted June 16, 1994) 


\section{Appendix}

\section{Linear propagation in a channel}

The channel longitudinal coordinate $x$ ranges from 0 to $L=16 \mathrm{~km}$. The disturbance does not depend on the transverse coordinate $y$, which makes the problem $1 \mathrm{D}$. The initial rise is given by:

$$
\begin{cases}\zeta_{0}(x)=\frac{1}{2} \cos \left[\frac{2 \pi}{\lambda}\left(x-\frac{L}{2}\right)\right]+\frac{1}{2} & x \in I \equiv\left[\frac{L}{2}-\frac{\lambda}{2}, \frac{\lambda}{2}+\frac{L}{2}\right] \\ \zeta_{0}(x)=0 & x \notin I\end{cases}
$$
while the initial velocity $u_{0}$ is zero. The bulge length $\lambda$ is $6 \mathrm{~km}$. Linear $1 \mathrm{D}$ theory gives the following
solution:

$$
\left\{\begin{array}{l}
\zeta(x, t)=\frac{1}{2}\left[\zeta_{0}(x-c t)+\zeta_{0}(x+c t)\right] \\
u(x, t)=\frac{g}{2 c}\left[\zeta_{0}(x-c t)-\zeta_{0}(x+c t)\right]
\end{array}\right.
$$

where $c=\sqrt{g h}$ is the constant wave phase velocity.

\section{Linear propagation in a rectangular basin}

The problem of a wave travelling in an axisymmetric basin, that is in a basin with depth depending only on the distance from a central point, admits a solution in terms of superposition of normal modes in the framework of the linear theory. If the basin is flat the solution is a combination of Bessel functions of simple argu-
ment. If the radial bottom profile is arbitrary, ment. If the radial bottom profile is arbitrary, then a method can be devised in order to compute the solution that is a piecewise combination of Bessel functions of real order and more complicated argument. This theory is treated in a paper by Tinti and Vannini (1994) and has been used here to calculate the theoretical solutions.
The initial perturbation is a bulge described by:

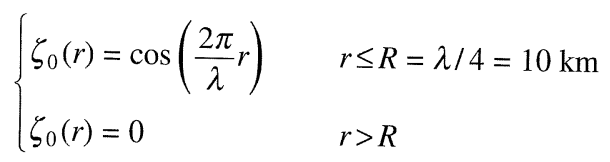
where $r$ is the distance from the mid-point of the right boundary of the rectangular basin. The corresponding
initial velocity is zero everywhere. 


\section{Nonlinear propagation in a channel}

The analytical solution of 1D trough travelling in a flat channel of length $L$ is computed by means of the theory of characteristics (Stoker, 1957). Let us assume that the initial fields are unperturbed, that is:

$$
\zeta_{0}(x)=u_{0}(x)=0
$$

and that the left end of the channel, corresponding to $x=0$, is excited by a prescribed forcing wave:

$$
\zeta(0, t)=c_{0} / g u(0, t)=\left\{\begin{array}{cc}
-2 \sin \left[\frac{2 \pi}{T} t\right] & t \leq T / 2 \\
0 & t \geq T / 2
\end{array}\right.
$$

during the time window $T=L / c_{0}$ where $c_{0}=\sqrt{g h}$. Under the above conditions one family of the characteristic curves is formed by straight lines in the quadrant $x \geq 0, t \geq 0$ of the plane $(x, t)$. On these curves the quantity

$$
u(x, t)-2 c(x, t)=-2 c_{0}
$$

where $c$ is the local phase velocity defined as:

$$
c(x, t)=\sqrt{g[h+\zeta(x, t)]}
$$

remains constant. Moreover, the curve slope depends on the forcing function $\zeta(0, t)$ as follows:

$$
\frac{d x}{d t}=3 c(0, t)-2 c_{0}
$$

Combining the above relations (A.5), (A.6) and (A.7), the solution can be computed in the channel for any time $t$ within the region of the plane $(x, t)$ where the characteristic straight lines do not intersect each other. 